\title{
PLASTIC ANISOTROPY PREDICTION BY ULTRASONIC TEXTURE DATA
}

\author{
V. N. SEREBRYANY \\ All-Russia Institute of Light Alloys, 121596, Moscow, Russia
}

(Received 20 June 1995)

\begin{abstract}
The plastic anisotropy parameters ( $R$ coefficient and height of ears of the drawn cup) have been calculated from ultrasonic orientation distribution function (ODF) coefficients on the basis of Taylor theory for low carbon steel and aluminium alloy sheets. The ODF coefficients were defined by Sayers method and using the iterative procedure on the basis of measurement of bulk longitudinal and shear wave time delays.
\end{abstract}

KEY WORDS: Ultrasonic bulk longitudinal and shear waves, ODF coefficients, $R$ coefficient, ears.

\section{INTRODUCTION}

The deep drawability of metal sheets used in the manufacture of car bodies or cans is estimated by the Lankford parameter $R$, i.e. the ratio of lateral to thickness strain. This parameter is defined mainly by the texture of the investigated metal sheet (Bunge, 1985 ). The mean value $\bar{R}$ is related to the limiting drawing ratio and the planar anisotropy $\Delta R$ is related to the height of ears of the drawn cup. These anisotropy parameters are usually obtained from mechanical destructive tests. Quite often, however, information on the texture obtained by nondestructive methods is required both for control of these parameters in the process of production of miscellaneous semifinished products and for predicting the texture and the associated anisotropy parameters. The ultrasonic method is one of these methods. It is based on the orientational dependence of the velocity of ultrasonic bulk waves of different types and polarizations (Sayers, 1982).

The objective of this paper is to devise a technique for ultrasonic nondestructive evaluation of the texture and plastic anisotropy parameters in stress-free metal sheets with the use of longitudinal and shear bulk waves.

\section{MATERIALS AND EXPERIMENTAL PROCEDURES}

This investigation was carried out on sheets of low carbon steel of grade $08 \mathrm{u}$ and the aluminium alloy of grade 3004 with thickness from 0.8 to $2.5 \mathrm{~mm}$. We used steel sheets in the delivered state after hot-cold rolling, annealing and skin rolling and aluminium alloy sheets after hot-cold rolling, annealing. The investigation of textures by X-ray diffraction analysis has been carried out by constructing direct and inverse pole figures. The method of inverse pole figures has been used for examining the heterogeneity of the texture across the sheet thickness. A layer-by layer investigation showed two types of texture across the sheet thickness in steel and aluminium alloy: 
a surface texture extending to $10 \%$ (for steel) and $20 \%$ (for aluminium alloy) of the sheet thickness at each side of a sheet and a core texture in the remaining $80 \%$ (for steel) and $60 \%$ (for aluminium alloy) of the sheet thickness. With account for the revealed texture heterogeneity across the sheet thickness, for the determination of the thickness-averaged texture by X-ray method, it is sufficient to obtain the direct pole figures for the surface and core layers. For the steel sheets the direct (110) and (200) pole figures were taken by the $\mathrm{X}$-ray reflection method using $\mathrm{CoK}_{\alpha}$ radiation. For the aluminium alloy sheets the direct (111) and (200) pole figures were taken by the same $\mathrm{X}$-ray method using $\mathrm{Cu} K_{\alpha}$ radiation. The thickness-averaged ODF coefficients have been calculated with account for the revealed texture heterogeneity from data on the direct pole figures by the series expansion method (Bunge, 1969) using the computer program "Texture Analyser". The error of the determination of X-ray ODF coefficients is not more than $10 \%$.

An ultrasonic investigation of the texture was carried out within the framework of Sayer's model (Sayers, 1982) by measuring the velocity of longitudinal and shear bulk waves. The method of combining the velocities of a longitudinal and two shear ultrasonic waves was used (Allen and Sayers, 1984). In this case the ultrasonic examination of the texture does not require measuring the density and thickness of sheets investigated, and the phase velocity of the ultrasonic waves can be judged by time delays, i.e., the time of ultrasonic wave propagation between the primary and the $n$-th reflected signal. Time delays were measured in an echo-pulse mode using acoustic piezoelectric transducers with a resonance frequency of $7-10 \mathrm{MHz}$. These transducers send a longitudinal and two shear ultrasonic waves in a direction normal to the sheet rolling plane, with the polarization planes of the waves oriented along and across the rolling direction, respectively. The instrumental error of the time delay measurements does not exceed 1 nsec. For each batch of a given metal, the time delays were measured at different points of a sheet. The number of measurements for each batch was not more than 45 .

\section{PREDICTION OF ANISOTROPY PARAMETERS}

\section{a. Estimation of the ODF coefficients}

(Sayers, 1982) based the method of the ODF coefficients determination on the solution of Christoffel's equation for the propagation of elastic waves in an anisotropic crystalline medium of rhombic symmetry. Instead of the elastic constants of single crystals he inserted in the equations the elastic constants of textured polycrystalline materials with a cubic lattice, which were calculated from elastic constants of single crystals averaged accordingly to the Reuss, Voigt, and Hill approximations with account for the weight function in the form of the ODF coefficients. In the absence of stresses, the sum of the square of the velocities of the longitudinal and two shear waves propagating in sheets with rhombic symmetry but having a cubic lattice does not depend on the texture of the metal investigated. This makes it possible to substitute in Sayers's equations the ratio of time delays squares of the waves for the squares of the velocities of the longitudinal and two shear waves and to exclude from the equations the density and thickness of the sheet. Then, having solved Sayer's equations for waves propagating along the normal to the sheet plane for the ODF coefficients, we get the formulae 
for the first two coefficients $C_{4}^{00}$ and $C_{4}^{02}$ in terms of time delays, elastic constants, and bulk compression and shear moduli (Serebryany, 1994):

$$
\begin{aligned}
& C_{4}^{00}=\frac{105 \sqrt{3}}{4 \sqrt{7} c}\left[\frac{B_{1} 1 / t_{1}^{2}}{\Sigma_{i=1} 1 / t_{i}^{2}}-B_{2}\right] \\
& C_{4}^{02}=\frac{105 \sqrt{3} B_{1}}{2 \sqrt{35} c \Sigma_{i=1} 1 / t_{i}^{2}}\left[\frac{1}{t_{3}^{2}} \cdot-\frac{1}{t_{2}^{2}}\right]
\end{aligned}
$$

where $t_{1}, t_{2}, t_{3}$ (in $\mu \mathrm{sec}$ ) are time of passage through the sheet thickness of a longitudinal wave and two shear waves polarized planes of which were orientated along and across the rolling direction; $B_{1}=K+10 \mu / 3 ; B_{2}=K+4 \mu / 3(K, \mu$ (in GPa) are bulk compression and shear moduli, calculated for polycrystalline isotropic materials by single crystal elastic constants); $c=C_{11}-C_{12}-C_{44}\left(C_{11}, C_{12}, C_{44}\right.$ are single crystal elastic constants, in $\mathrm{GPa}$ ). The higher order coefficients can be defined in terms of ideal texture components with Gaussian spread around the orientations and their volume fractions as follows:

$$
C_{l}^{\mu v}=\sum_{i=1}^{n} V_{i}(g) C_{l}^{\mu \nu}(g)
$$

Here, $n, V_{i}(g)$, and $C_{l}^{\mu v}(g)$ are the number of orientations $g$, their individual volume fractions, and their ODF coefficients, respectively. In turn, the volume fractions of the designed ideal orientations of the investigated metal can be defined, if we solve Equations (3) for $V_{i}(g)$ by a least-squares method with using an iterative procedure, provided that instead of the $C_{l}^{\mu \nu}$ coefficients we inserted in the equations the $C_{4}^{00}$ and $C_{4}^{02}$ coefficients, obtained from Equations (1), (2).

\section{b. Calculation of the anisotropy parameters}

The ultrasonic ODF coefficients have been used for calculation of $x$ - angle dependence of the Taylor factor $\bar{M}(q, x)$, which characterizes the parameters of sheet plane and normal anisotropty (Bunge, 1985):

$$
\bar{M}(q, x)=\sum_{l=0}^{6} \sum_{n=0}^{l} \sum_{\rho=0}^{N} \frac{m_{l \rho}^{0 n} C_{l}^{0 n} \cos n x q^{\rho}}{2 l+1}
$$

Here, $m_{l \rho}^{0 n}$ are coefficients of expansion into a series of sherical functions and power series of $q$ of the orientation dependence of the Taylor factor for single crystals, calculated on the basis of Taylor plastic deformation model; $q$-parameter of strain tensor, conforming to tensile strain conditions of the plane speciments of investigated sheets. At the given $x$-angle, the $R(x)$ value can be calculated using $\bar{M}(q, x)$

$$
R(x)=\frac{q_{\min }(x)}{1-q_{\min }(x)}
$$

Here, $q_{\min }(x)$ are values of $q$, at which $\bar{M}(q, x)$ achieves its minimal value for the given $x$-angle. We obtained the linear regression relationship between height $(h)$ of the ears and calculated, in view of Equations (4), (5), parameter $\Delta R$ using a least-squares 
method, as applied to aluminium alloy sheets (Serebryany, 1995). Using this relationship we can predict the distribution of ears in the drawn cup as a result of the deep drawing of a circular blank cut from aluminium sheet into a cylindrical cup.

\section{RESULTS AND DISCUSSION}

Ultrasonic ODF coefficients for steel sheets have been defined in view of Equations (1) - (3). The calculation was made on the basis of the published results (Serebryany, 1994), i.e. by using the elastic constants $C_{11}=237 \mathrm{GPa}, C_{12}=141 \mathrm{GPa}$, and $C_{44}=116 \mathrm{GPa}$ and the averaging of these constants in Reuss approximation. For the definition of the volume fractions of the orientations we selected the six principal orientations (554)[225], (665)[110], (111)[231], (112)[110], (001)[110], (111)[011], all of which are important in cold-rolled and annealed low carbon steel sheets and are widely separated from one another. The ultrasonic and X-ray ODF coefficients of the investigated steel sheet are listed in Table 1.

The results presented in Table 1 show that for steel sheets the ultrasonic ODF coefficients agree rather well within the limits of experimental errors with the X-ray ODF coefficients. These ODF coefficients were used for the calculation of the $R$ parameter in view of Equations (4), (5). The $R(x)$ - function is defined, provided that tensile deformation of the low carbon steel was supposed to be caused by (hkl)[111]

Table 1 Ultrasonic and X-ray coefficients for the low carbon steel and aluminium alloy sheets

\begin{tabular}{lcccc}
\hline & \multicolumn{2}{c}{ Steel } & \multicolumn{2}{c}{ Aluminium } \\
$C_{l}^{0 v}$ & Ultr. & X-ray & Ultr. & X-ray \\
\hline$C_{4}^{00}$ & -1.15 & -1.02 & 0.95 & 0.82 \\
$C_{4}^{02}$ & -0.30 & -0.49 & 0.07 & 0.04 \\
$C_{4}^{04}$ & 0.22 & 0.18 & 0.50 & 0.53 \\
$C_{6}^{00}$ & 1.70 & 2.32 & 0.50 & 0.43 \\
$C_{6}^{02}$ & -0.23 & -1.42 & 0.48 & 0.10 \\
$C_{6}^{04}$ & 0.35 & 0.47 & -1.22 & -1.32 \\
\hline
\end{tabular}

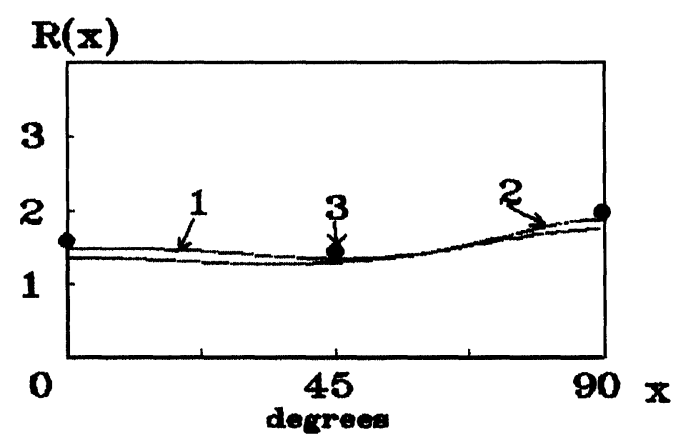

Figure $1 R(x)$ - values defined from ultrasonic (1), X-ray (2) and mechanical (3) data for steel sheet. 
glide systems ('pensil' glide). The calculated $R$-parameters based on the ultrasonic experiment are compared to corresponding parameters, obtained from mechanical tests results and calculations using X-ray coefficients of ODF. These planar $R$-value distributions of the low carbon steel sheets are presented in Figure 1. Ultrasonic values of $R(x)$ correlate within the limits of experimental errors with $\mathrm{X}$-ray values and with values, obtained from mechanical tests of specimens cut at the different $x$-angles to rolling direction.

Ultrasonic ODF coefficients for aluminium alloy sheets have been defined in view of Equations (1)-(3) by using the elastic constants $C_{11}=108.2 \mathrm{GPa}, C_{12}=61.3 \mathrm{GPa}$, $C_{44}=28.5 \mathrm{GPa}$ and the averaging of these constants in Voigt approximation. The four principal orientations (001)[100], (131)[323], (427)[232], (120)[001] were selected for the evaluation of the volume fractions of the orientations. These orientations defined mainly the texture of the investigated alloy sheets. The ultrasonic and X-ray ODF coefficients of the aluminium alloy sheet are listed in Table 1. The results presented in Table 1 show that for the given aluminium alloy sheet the ultrasonic ODF coefficients agree rather well within the limits of experimental errors with the X-ray ones. These ODF coefficients were used for the evaluation of the ears position and height in the drawn cups according to the technique (Serebryany, 1995). The $h$-parameters are defined provided that tensile deformation of the given aluminium alloy was supposed to be caused by (111)[110] glide systems. The earing distributions calculated on the basis of the ultrasonic and X-ray experiments are presented in Figure 2. Ultrasonic values of $h(x)$ correlate within the limits of experimental errors with X-ray ones.

\section{CONCLUSIONS}

The $R$ and $h$ values predictions based on the ultrasonic ODF coefficients up to $1=6$, obtained in this study, lead to rather well agreement with tensile test and $\mathrm{X}$-ray results.

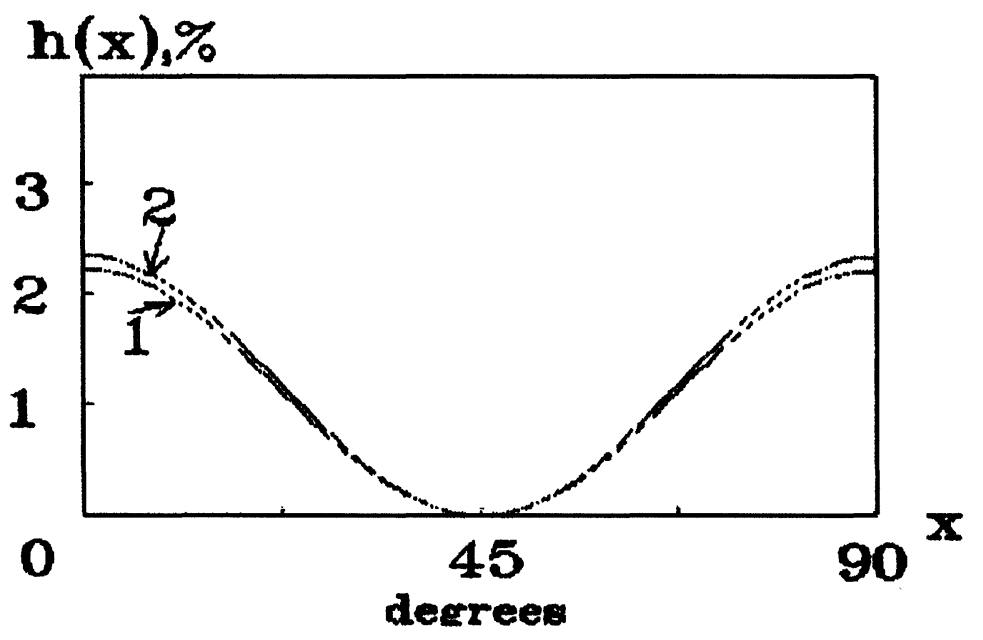

Figure $2 h(x)$ - values defined from ultrasonic (1) and X-ray (2) data for aluminium sheet. 


\section{Acknowledgements}

The author expresses his thanks to Dr. V. Slavov (JSC 'North-steel', Cherepovets) for carrying out the X-ray tests and to Dr. S. Kurtasov (Institute of Geosystems, Moscow) for the access to the program "Texture Analyser".

\section{References}

Allen, D. R. and Sayers, C. M. (1984). The measurement of residual stress in textured steel using an ultrasonic velocity combinations technique, Ultrasonics, 22(4), 179-188.

Bunge, H. J. (1969). Mathematical Methods of Texture Analysis [in German], Akademie Verlag, Berlin.

Bunge, H. J. (1985). Technological applications of texture analysis, Z. Metallkde., 76, 457-470.

Sayer, C. M. (1982). Ultrasonic velocities in anisotropic polycrystalline aggregates, J. Phys. D.: Appl. Phys., 15, 2157-2167.

Serebryany, V. N. (1994). Quantitative estimation of steel sheet texture by method of ultrasonic bulk waves, Ind. Lab., 60(4), 223-227.

Serebryany, V. N. (1995). Earing evaluation during deep drawing in aluminium alloys [in Russian]. Zav. Lab., 61(3), 15-18. 